Surveys in Differential Geometry XV

\title{
The Signature of the Seiberg-Witten Surface
}

\author{
Andreas Malmendier
}

\begin{abstract}
The Seiberg-Witten family of elliptic curves defines a Jacobian rational elliptic surface $\mathrm{Z}$ over $\mathbb{C P}^{1}$. We show that for the $\bar{\partial}$-operator along the fiber the logarithm of the regularized determinant $-\frac{1}{2} \log \operatorname{det}^{\prime}\left(\bar{\partial}^{*} \bar{\partial}\right)$ satisfies the anomaly equation of the oneloop topological string amplitude derived in Kodaira-Spencer theory. We also show that not only the determinant line bundle with the Quillen metric but also the $\bar{\partial}$-operator itself extends across the nodal fibers of $\mathrm{Z}$. The extension introduces current contributions to the curvature of the determinant line bundle at the points where the fibration develops nodal fibers. The global anomaly of the determinant line bundle then determines the signature of $\mathrm{Z}$ which equals minus the number of hypermultiplets.
\end{abstract}

\section{Introduction and Statement of results}

There has been a continuing interest in the non-perturbative properties of the supersymmetric Yang-Mills theory on four-dimensional manifolds. One of the results of the work of Seiberg and Witten [19] is that the moduli space of the topological $S U(2)$-Yang-Mills theory on a fourdimensional manifold decomposes into two branches, the Coulomb branch and the Seiberg-Witten branch. The branches are interpreted as the moduli spaces of simpler physical theories on the four-manifold. The Coulomb branch, also called the Seiberg-Witten family of curves, is the moduli space of a topological $U(1)$-gauge theory, called the low energy effective $U(1)$ gauge theory. We investigate the geometry and topology of the Coulomb branch as it is fundamental for the definition and the understanding of the $N=2$ supersymmetric, low energy effective field theory by using the results and techniques developed in $[\mathbf{2}, \mathbf{1 8}, \mathbf{5}, \mathbf{6}]$.

This article is structured as follows: In Section 2 we explain how the Seiberg-Witten families of elliptic curves for the $N=2$ supersymmetric $S U(2)$-gauge theory with $N_{f}=0,1,2,3,4$ additional fields, called

(C)2011 International Press 
hypermultiplets, define four-dimensional, Jacobian rational elliptic surfaces $\overline{\mathrm{Z}} \rightarrow \mathbb{C P}^{1}$ with singular fibers. We describe the correspondence between the rational elliptic surfaces and the constraints on the masses of the hypermultiplets. Unless noted otherwise, we will then always assume that the masses are generic so that any singular fiber over $\left[u^{*}: 1\right] \in \mathbb{C P}{ }^{1}$ for finite $u^{*}$ is a node and does not give rise to a surface singularity. Only the singular fiber over $[1: 0]$ is a cusp giving rise to a surface singularity. Thus, after removing the singular fiber over $[1: 0]$, we obtain a smooth elliptic surface $\mathrm{Z}$ with three-dimensional boundary and elliptically fibered over a bounded disc in $\mathbb{C P}^{1}$, called the $u$-plane $\mathrm{UP} \subset \mathbb{C P}^{1}$.

In Section 3 we review the construction of the regularized determinant of the $\bar{\partial}$-operator on an elliptic curve. When the elliptic curve $E_{u}$ is varied in the Seiberg-Witten family over the $u$-plane, we obtain the determinant line bundle DET $\bar{\partial} \rightarrow$ UP of the $\bar{\partial}$-operator along the fiber of $\mathrm{Z} \rightarrow \mathrm{UP}$. The regularized determinant $\operatorname{det}^{\prime}\left(\bar{\partial}^{*} \bar{\partial}\right)$ of the Laplacian along the fiber becomes a smooth function on UP which we will later use in the Quillen construction to define a metric and connection on DET $\bar{\partial}$. In Section 4 , we show that the logarithm of the regularized determinant of the Laplacian $-\frac{1}{2} \ln \operatorname{det}^{\prime}\left(\bar{\partial}^{*} \bar{\partial}\right)$ satisfies the anomaly equation for the one-loop topological string amplitude of Kodaira-Spencer theory derived in [4].

In Section 5 we show that the local anomaly of DET $\bar{\partial}$ vanishes and determine the non-trivial global anomaly as holonomy of the determinant section. We use the results of Bismut and Bost $[\mathbf{5}, \mathbf{6}]$ to show that the determinant line bundle with the Quillen metric extends smoothly across the nodal fibers. Because of the non-trivial holonomy the extension of the determinant line bundle introduces current contributions to the curvature over the points in the $u$-plane where the fiber develops a node.

In the case that the singular fibers are nodes Seeley and Singer [18] showed that there is a $\bar{\partial}$-operator that is defined on the nodal fiber as well so that in a neighborhood $U \subset \mathrm{UP}$ the family of operators $\left\{\bar{\partial}_{u}\right\}_{u \in U}$ is a continuous family. However, their procedure of obtaining the determinant line bundle is different from Bismut and Bost $[\mathbf{5 , 6}]$ because their Laplacian is different. In Section 6 we explain the connection between the two procedures by a local change in the conformal gauge of the fiber metric in a neighborhood of the nodal fiber.

In Section 7 and Section 8 we discuss the elliptic operators connected to the signature of the elliptic surface $\mathrm{Z} \rightarrow \mathrm{UP}$. First, for the signature operator $\mathrm{D}$ along the fiber of $\mathrm{Z} \rightarrow \mathrm{UP}$ we compute the global anomaly. We show that there is a canonical trivialization of the determinant line bundle (DET D) ${ }^{\otimes 6}$, and the well-defined logarithmic monodromies of the canonical section of DET D determine the signature of $\mathrm{Z} \rightarrow \mathrm{UP}$. We interpret the determinant line bundle as a solution to a Riemann-Hilbert problem on $\mathbb{C P}^{1}$. On the other hand, the generalization of Hirzebruch's signature theorem for manifolds with boundary by Atiyah, Patodi, Singer (APS) [3] shows that the elliptic signature operator on the four-dimensional surface $\mathrm{Z}$ has an analytic index 
if one imposes APS boundary conditions on $\partial \mathrm{Z}$. We show that this analytic index equals minus the number $N_{f}$ of hypermultiplets.

\section{The Jacobian elliptic surfaces for $N=2$ Yang-Mills theory}

An elliptic curve $E$ in the Weierstrass form can be written as

$$
y^{2}=4 x^{3}-g_{2} x-g_{3},
$$

where $g_{2}$ and $g_{3}$ are numbers such that the discriminant $\Delta=g_{2}^{3}-27 g_{3}$ does not vanish. In homogeneous coordinates $[X: Y: W]$ Eq. (2.1) becomes

$$
W Y^{2}=4 X^{3}-g_{2} X W^{2}-g_{3} W^{3} .
$$

One can check that the point $P$ with coordinates $[0: 1: 0]$ is a smooth point of the curve. We consider $P$ the base point of the elliptic curve and the origin of the group law on $E$. The two types of singularities that can occur as Weierstrass cubic are a rational curve with a node, which appears when the discriminant vanishes and $g_{2}, g_{3} \neq 0$, or a cusp when $g_{2}=g_{3}=0$.

Next, we look at a family of cubic curves over $\mathbb{C P}^{1}$. The family is parametrized by the base space $\mathbb{C P}^{1}$ and a line bundle $\mathcal{N} \rightarrow \mathbb{C P}^{1}$. The quantities $g_{2}$ and $g_{3}$ are promoted to global sections of $\mathcal{N}^{\otimes 4}$ and $\mathcal{N}^{\otimes 6}$ respectively; the discriminant becomes a section of $\mathcal{N}^{\otimes 12}$. If the sections are generic enough so that they do not always lie in the discriminant locus, we obtain a Weierstrass fibration $\pi: \overline{\mathrm{Z}} \rightarrow \mathbb{C P}^{1}$ with a section, called a Jacobian elliptic fibration. Each fiber comes equipped with the base point $P$ that defines a section $S$ of the elliptic fibration which does not pass through the nodes or cusps. We will always assume that $\mathcal{N}=\mathcal{O}_{\mathbb{C P}^{1}}(-1)$.

In addition, we will assume from now on that in the coordinate chart $[u: 1] \in \mathbb{C P}^{1}$, the discriminant $\Delta$ is a polynomial of degree $2 \leq N_{f}+2 \leq 6$ in $u$ where $0 \leq N_{f} \leq 4$, and $g_{2}$ and $g_{3}$ are polynomials in $u$ of degree at most 2 and 3 respectively. The space of all such Weierstrass elliptic surfaces has $N_{f}+1$ moduli. To see this consider first the case where $N_{f}=4$. From the seven parameters defining $g_{2}$ and $g_{3}$ two can be eliminated by scaling and a shift in $u$. Furthermore, we can arrange the coefficient of $g_{2}$ of degree two and the coefficient of $g_{3}$ of degree three to be the modular invariants of an elliptic curve with periods 1 and $\tau_{0}$. The remaining four coefficients can be expressed in terms of four complex parameters. Following the convention of $[\mathbf{1 9}]$ we will denote the parameters by $m_{1}, \ldots, m_{N_{f}}$. In physics, they are called the masses of the hypermultiplets.

A non-trivial elliptic fibration has to develop singular fibers. The classification of the singular fibers is part of Kodaira's classification theorem of all possible singular fibers of an elliptic fibration [9]. For generic values of the masses, the polynomial $\Delta$ has $N_{f}+2$ simple zeros $u_{1}^{*}, \ldots, u_{N_{f}+2}^{*}$ for $|u|<\infty$ with $g_{2}\left(u_{i}^{*}\right), g_{3}\left(u_{i}^{*}\right) \neq 0$ for $i=1, \ldots, N_{f}+2$. From Kodaira's classification theorem of singular fibers $[\mathbf{9}]$ it follows that the elliptic fibration develops the nodes, i.e., a singular fibers of Kodaira type $I_{1}$ over the points 
$u_{1}^{*}, u_{2}^{*}, \ldots, u_{N_{f}+2}^{*}$. For special values for $m_{1}, \ldots, m_{N_{f}}$, several singular fibers of Kodaira type $I_{1}$ can coalesce and form singular fibers of Kodaira type $I_{k}$ with $k \geq 2$, where the discriminant has a zero of order $k$. The second chart over the base space is $[1: v] \in \mathbb{C P}^{1}$. The intersection of the two charts is given by $u=1 / v$ with $v \neq 0$. The Weierstrass coordinates transform according to $x \mapsto v^{2} x$ and $y \mapsto v^{3} y$; since $g_{2}$ and $g_{3}$ are sections of $\mathcal{N}^{4}$ and $\mathcal{N}^{6}$ respectively, they transform according to $g_{2} \mapsto v^{4} g_{2}$ and $g_{3} \mapsto v^{6} g_{3}$. The discriminant $\boldsymbol{\Delta} \mapsto v^{12} \boldsymbol{\Delta}$ becomes a polynomial in $v$ of degree $10-N_{f}$. From Kodaira's classification theorem it follows that the singular fiber $E_{\infty}$ over $u=\infty(v=0)$ is a cusp, a singular fiber of Kodaira type $I_{4-N_{f}}^{*}$.

REMARK 1. Under the change of the coordinate chart from $[u: 1]$ to $[1: v]$ on $\mathbb{C P}^{1}$ by $u=-1 / v$ with $v \neq 0$ the holomorphic one-form $d z=d x / y$ transforms as

$$
d z_{u}=\frac{d x_{u}}{y_{u}}=-v \frac{d x_{v}}{y_{v}}=-v d z_{v} .
$$

It follows that $(d z)^{\otimes 2}$ has the same transformation under a coordinate change as $(d u)^{-1}$ whence $\mathcal{N}^{2} \cong \omega_{\mathbb{C P}^{1}}^{-1}$ where $\omega_{\mathbb{C P}^{1}}$ is the canonical bundle on $\mathbb{C P}^{1}$.

The elliptic surface $\bar{Z}$ is a hyper-surface in the variables $(u,[X: Y: W])$. $\bar{Z}$ has surface singular points whenever all partial derivatives in $u, x, y$ simultaneously vanish. Singular fibers of Kodaira type $I_{1}$ do not give rise to surface singularities, whereas all singular fibers of Kodaira type $I_{n}$, with $n \geq 2$, and $I_{n}^{*}$, with $n \geq 0$, do. The monodromy around singular fibers of type $I_{n}$ or $I_{n}^{*}$ is parabolic [9]. It is known [12, Sec. 4.6] that a Weierstrass fibration is rational (i.e., birational to $\mathbb{C P}^{2}$ ), if $g_{2}$ and $g_{3}$ are polynomials in $u$ of degree at most 4 and 6 respectively. The minimal resolution $Z$ of $\bar{Z}$ is the blow-up of $\mathbb{C P}^{2}$ in nine points, and therefore has Picard number 10 . Conversely, by contracting every component of the fiber which does not meet $S$, we obtain back the normal surface $\bar{Z}$. The section $S$ and smooth fiber use up two dimensions, and so the number of components of any singular fiber is at most eight since the components are always independent in the Neron-Severi group. However, as we will see below not all configurations of singular fibers exist.

For later use we also introduce the following notation: we will denote by UP the base curve $\mathbb{C P}^{1}$ minus a small disc around $u=\infty$, and the restrition of the elliptic fibration to UP will be denoted by $\mathrm{Z} \rightarrow \mathrm{UP}$. Similarly, UP will denote the base curve $\mathbb{C P}^{1}$ with small open discs around all points with singular fibers removed, and the restriction of the elliptic fibration to $\stackrel{\circ}{\mathrm{U}}$ will be denoted by $\stackrel{\circ}{\mathrm{Z}} \rightarrow \stackrel{\circ}{\mathrm{UP}}$.

REMARK 2. We denote by $\omega_{Z}$ the canonical bundle of $Z$. The space $H^{0}\left(\omega_{\mathrm{Z}}\right)$ is the space of global holomorphic two-forms of dimension 
$p_{g}=h^{2,0}$. The bundle $\omega_{\mathrm{Z} / \mathbb{C P}^{1}}=\omega_{\mathrm{Z}} \otimes\left(\pi^{*} \omega_{\mathbb{C P}^{1}}\right)^{-1}$ restricts to the canonical line bundle $K_{u}=\omega_{E_{u}}$ on each smooth fiber $E_{u}$. It is well-know that for any rational elliptic surface we have $h^{2,0}=h^{1,0}=0[\mathbf{1 2}$, Thm. 2.10] and the canonical class is minus the fiber class. Hence, we have $c_{1}^{2}(\mathrm{Z})=0$. It also follows that the first Chern class $c_{1}\left(\omega_{\mathrm{Z} / \mathbb{C P}^{1}}\right)$ is a pullback from the base manifold and $c_{1}^{2}\left(\omega_{\mathrm{Z} / \mathbb{C P}^{1}}\right)=0$.

The above discussion motivates the following definition:

Definition 2.1. A Seiberg-Witten curve for $N_{f}$ hypermultiplets is a Jacobian rational elliptic surface with one singular fiber of Kodaira type $I_{4-N_{f}}^{*}$ and singular fibers of Kodaira type $I_{n}$ and $I_{n}^{*}$ only.

Using the explicit Weierstrass parametrization given in [19] for $0 \leq$ $N_{f} \leq 4$ it is easy to compose a list of the possible configurations of singular fibers that appear as Seiberg-Witten curves. In Table 1, we list the constraints on the moduli, which substituted into the Weierstrass presentation in $[\mathbf{1 9}]$ realize the configuration of singular fibers, the structure of the singular fibers $E_{u_{n}^{*}}$ over finite $u_{n}^{*}$, and $E_{\infty}$ for the rational elliptic surface $\overline{\mathrm{Z}} \rightarrow \mathbb{C P}^{1}$. Here, $r$ is given by $r=8-\sum_{\nu}\left(m_{\nu}-1\right)$ where the sum in $\nu$ runs over all singular fibers of the elliptic surface, and $m_{\nu}$ denotes the number of irreducible components in the singular fiber.

\section{TABLE 1}

\begin{tabular}{ccccl}
\hline$N_{f}$ & $r$ & $E_{\infty}$ & singular fibers $E_{u_{n}^{*}}$ & mass constraints \\
\hline 4 & 4 & $I_{0}^{*}$ & $6 I_{1}$ & - \\
4 & 3 & $I_{0}^{*}$ & $I_{2}, 4 I_{1}$ & $m_{3}=m_{4} \neq 0$ \\
4 & 2 & $I_{0}^{*}$ & $I_{3}, 3 I_{1}$ & $m_{2}=m_{3}=m_{4} \neq 0$ \\
4 & 2 & $I_{0}^{*}$ & $2 I_{2}, 2 I_{1}$ & $m_{3}=m_{4}=0$ \\
4 & 1 & $I_{0}^{*}$ & $I_{4}, 2 I_{1}$ & $m_{2}=m_{3}=m_{4}=0$ \\
4 & 1 & $I_{0}^{*}$ & $3 I_{2}$ & $m_{1}=m_{2}, m_{3}=m_{4}=0$ \\
4 & 0 & $I_{0}^{*}$ & $I_{0}^{*}$ & $m_{1}=m_{2}=m_{3}=m_{4}=0$ \\
3 & 3 & $I_{1}^{*}$ & $5 I_{1}$ & - \\
3 & 2 & $I_{1}^{*}$ & $I_{2}, 3 I_{1}$ & $m_{2}=m_{3}$ \\
3 & 1 & $I_{1}^{*}$ & $I_{3}, 2 I_{1}$ & $m_{1}=m_{2}=m_{3} \neq 0$ \\
3 & 1 & $I_{1}^{*}$ & $2 I_{2}, I_{1}$ & $m_{1}=m_{2}=0$ \\
3 & 0 & $I_{1}^{*}$ & $I_{4}, I_{1}$ & $m_{1}=m_{2}=m_{3}=0$ \\
2 & 2 & $I_{2}^{*}$ & $4 I_{1}$ & - \\
2 & 1 & $I_{2}^{*}$ & $I_{2}, 2 I_{1}$ & $m_{1}=m_{2} \neq 0$ \\
2 & 0 & $I_{2}^{*}$ & $2 I_{2}$ & $m_{1}=m_{2}=0$ \\
1 & 1 & $I_{3}^{*}$ & $3 I_{1}$ & - \\
0 & 0 & $I_{4}^{*}$ & $2 I_{1}$ & - \\
\hline
\end{tabular}


REMARK. The completeness of Table 1 follows by comparing the list with the list of impossible configurations in [11]. It follows from [14] that among the Seiberg-Witten curves the ones with $r=0$ are the only modular elliptic surfaces.

\section{The regularized determinant on an elliptic curve}

We consider an elliptic curve $E$ with periods $2 \omega$ and $2 \omega^{\prime}$, modular parameter $\tau=\frac{\omega^{\prime}}{\boldsymbol{\omega}}$, and complex coordinate $z$. Let $\xi=\xi^{1}+i \xi^{2}$ be the complex coordinate on the normalized torus with periods 1 and $\tau$ such that $\xi=\frac{z}{2 \boldsymbol{\omega}}$. For $n_{1}, n_{2} \in \mathbb{N}$, a complex function $\varphi$ on the normalized torus with the periodicities

$$
\begin{aligned}
\varphi\left(\xi^{1}+1, \xi^{2}\right) & =-e^{\pi i \nu_{1}} \varphi\left(\xi^{1}, \xi^{2}\right), \\
\varphi\left(\xi^{1}+\operatorname{Re} \tau, \xi^{2}+\operatorname{Im} \tau\right) & =-e^{\pi i \nu_{2}} \varphi\left(\xi^{1}, \xi^{2}\right),
\end{aligned}
$$

is given by

$$
\begin{aligned}
\varphi_{n_{1}, n_{2}}\left(\xi^{1}, \xi^{2}\right)= & \exp 2 \pi i\left\{\left[n_{1}+\frac{1-\nu_{1}}{2}\right] \xi^{1}\right. \\
& \left.+\frac{1}{\operatorname{Im} \tau}\left[n_{2}+\frac{1-\nu_{2}}{2}-\operatorname{Re} \tau\left(n_{1}+\frac{1-\nu_{1}}{2}\right)\right] \xi^{2}\right\} .
\end{aligned}
$$

In fact, the set of functions $\left\{\varphi_{n_{1}, n_{2}}\right\}$ constitutes a complete system of eigenfunctions for the Laplace operator $-4 \partial_{\xi} \bar{\partial}_{\xi}$ where $2 \bar{\partial}_{\xi}=\partial_{\xi^{1}}+i \partial_{\xi^{2}}$. Their eigenvalues under $2 \bar{\partial}_{\xi}$ are

$$
\frac{2 \pi}{\operatorname{Im} \tau}\left\{\left(n_{1}+\frac{1-\nu_{1}}{2}\right) \tau-\left(n_{2}+\frac{1-\nu_{2}}{2}\right)\right\} .
$$

Because of $2 \bar{\partial}=2 \bar{\partial}_{z}=\frac{1}{\bar{\omega}} \bar{\partial}_{\xi}$ the functions $\varphi_{n_{1}, n_{2}}$ are also eigenfunctions of $2 \bar{\partial}$ for the eigenvalues

$$
\frac{\pi}{\operatorname{Im} \tau \bar{\omega}}\left\{\left(n_{1}+\frac{1-\nu_{1}}{2}\right) \tau-\left(n_{2}+\frac{1-\nu_{2}}{2}\right)\right\} .
$$

The holomorphic line bundle of positive spinors on an elliptic curve $E$ can also be interpreted as a holomorphic square root $K^{1 / 2}$ of the bundle of holomorphic $(1,0)$-forms $K=\Omega^{1,0}(E)$. The chiral Dirac operators are

$$
\begin{gathered}
\not \partial^{+}=\bar{\partial}: C^{\infty}\left(K^{1 / 2}\right) \rightarrow C^{\infty}\left(K^{1 / 2} \otimes \bar{K}\right), \\
\not \partial^{-}=-\partial: C^{\infty}\left(\overline{K^{1 / 2}}\right) \rightarrow C^{\infty}\left(\overline{K^{1 / 2}} \otimes K\right) .
\end{gathered}
$$

Equivalently, we can view the situation as follows: we choose the unique even spin structure on $E$ as a reference square root $K_{0}^{1 / 2} \cdot K_{0}^{1 / 2}$ is the preferred spin bundle for the chosen homology basis, its divisor $\kappa=1 / 2+\tau / 2$ is the vector of Riemann constants. If we twist the Dirac operator $\not^{+}$by a flat 
holomorphic line bundle $W_{\left(\nu_{1}, \nu_{2}\right)}$ of order two with divisor $\check{\xi}=-\frac{\nu_{2}}{2}-\frac{\nu_{1}}{2} \tau$ and $\nu_{1}, \nu_{2} \in\{0,1\}$, the twisted chiral Dirac operator becomes

$$
\not \partial_{\left(\nu_{1}, \nu_{2}\right)}^{+}=\bar{\partial}_{\left(\nu_{1}, \nu_{2}\right)}: C^{\infty}\left(K_{0}^{1 / 2} \otimes W_{\left(\nu_{1}, \nu_{2}\right)}\right) \rightarrow C^{\infty}\left(K_{0}^{1 / 2} \otimes W_{\left(\nu_{1}, \nu_{2}\right)} \otimes \bar{K}\right) .
$$

In other words, the twisted chiral Dirac operator is the $\bar{\partial}$-operator coupled to the holomorphic line bundle $L=K_{0}^{1 / 2} \otimes W_{\left(\nu_{1}, \nu_{2}\right)}$. The functions

$$
\varphi_{n_{1}, n_{2}}^{+}(z)=\sqrt{d z} \varphi_{n_{1}, n_{2}}(z) \in C^{\infty}\left(K_{0}^{1 / 2} \otimes W_{\left(\nu_{1}, \nu_{2}\right)}\right)
$$

form a complete system of eigenfunctions for the operator $(-4 \partial \bar{\partial})_{\left(\nu_{1}, \nu_{2}\right)}$ with the eigenvalues

$$
\left(\frac{\pi}{\operatorname{Im} \tau|\boldsymbol{\omega}|}\right)^{2}\left|\left(n_{1}+\frac{1-\nu_{1}}{2}\right) \tau-\left(n_{2}+\frac{1-\nu_{2}}{2}\right)\right|^{2} .
$$

Using the Kähler form one can identify $\sqrt{d z} \otimes d \bar{z}$ with $\sqrt{d \bar{z}}$. The $\zeta$-function (3.3)

$$
\zeta_{\left(\nu_{1}, \nu_{2}\right)}(s)=\sum_{n_{1}, n_{2}} \frac{1}{\left[\left(n_{1}+\frac{1-\nu_{1}}{2}\right)^{2} \operatorname{Im}^{2} \tau+\left(\left(n_{1}+\frac{1-\nu_{1}}{2}\right) \operatorname{Re} \tau-\left(n_{2}+\frac{1-\nu_{2}}{2}\right)\right)^{2}\right]^{s}}
$$

is absolutely convergent for $\operatorname{Re} s>1$. When $\left(\nu_{1}, \nu_{2}\right)=(1,1)$ it is understood that the summation does not include $n_{1}=n_{2}=0$. The function $\zeta_{\left(\nu_{1}, \nu_{2}\right)}$ is well-defined and has a meromorphic extension to $\mathbb{C}$ and 0 is not a pole. The regularized determinant of $-4 \partial \bar{\partial}$ is defined by setting

$$
\ln \operatorname{det}(-4 \partial \bar{\partial})_{\left(\nu_{1}, \nu_{2}\right)}:=-\left[\frac{1}{\left(\frac{\pi}{\operatorname{Im} \tau|\boldsymbol{\omega}|}\right)^{2 s}} \zeta_{\left(\nu_{1}, \nu_{2}\right)}(s)\right]^{\prime}
$$

It follows that

$$
\ln \operatorname{det}(-4 \partial \bar{\partial})_{\left(\nu_{1}, \nu_{2}\right)}=-\zeta_{\left(\nu_{1}, \nu_{2}\right)}^{\prime}(0)+\ln \left(\frac{\pi}{\operatorname{Im} \tau|\boldsymbol{\omega}|}\right)^{2} \zeta_{\left(\nu_{1}, \nu_{2}\right)}(0) .
$$

It was shown in $[\mathbf{1 7}]$ that $\zeta(0)=0$ for $\left(\nu_{1}, \nu_{2}\right) \neq(1,1)$, and $\zeta(0)=-1$ for $\left(\nu_{1}, \nu_{2}\right)=(1,1)$. It follows that

$$
\begin{aligned}
\operatorname{det}^{\prime}(-4 \partial \bar{\partial})_{(1,1)} & =\frac{4 \operatorname{Im}^{2}(\tau)|\boldsymbol{\omega}|^{2}}{(2 \pi)^{2}}|\boldsymbol{\eta}(\tau)|^{4} \\
\operatorname{det}(-4 \partial \bar{\partial})_{\left(\nu_{1}, \nu_{2}\right)} & =\left|\frac{\vartheta_{\nu_{1} \nu_{2}}(\tau)}{\boldsymbol{\eta}(\tau)}\right|^{2}=e^{-\frac{2 \pi}{\operatorname{Im} \tau}(\operatorname{Im} \check{\xi})^{2}}\left|\frac{\vartheta(\check{\xi} \mid \tau)}{\boldsymbol{\eta}(\tau)}\right|^{2},
\end{aligned}
$$


where the Dedekind $\boldsymbol{\eta}$-function and the Jacobi $\vartheta$-function $\vartheta(v \mid \tau)=\vartheta_{00}(v \mid \tau)$ are given by

$$
\begin{aligned}
\boldsymbol{\eta}(\tau) & =e^{\frac{\pi i \tau}{12}} \prod_{n=1}^{\infty}\left(1-e^{2 \pi i n \tau}\right) \\
\vartheta_{a b}(v \mid \tau) & =\sum_{n \in \mathbb{Z}} \exp \left[i \pi\left(n+\frac{a}{2}\right)^{2} \tau+2 \pi i\left(n+\frac{a}{2}\right)\left(v+\frac{b}{2}\right)\right] .
\end{aligned}
$$

\section{The topological one-loop string amplitude}

For each smooth fiber $E_{u}$ of the fibration $\mathrm{Z} \rightarrow \mathrm{UP}$ we have dim $H^{1}\left(E_{u}\right)=2$. Since a base point is given in each fiber by the section $S$, we can choose a symplectic basis $\left\{\alpha_{u}, \beta_{u}\right\}$ of the homology $H_{1}\left(E_{u}\right)$ with respect to the intersection form, called a homological marking consisting of the A-cycle and B-cycle. We cannot define $\alpha_{u}, \beta_{u}$ globally over UP. The cycles are transformed by monodromies around the points with singular fibers. However, we can define globally an analytical marking. An analytical marking is a choice of a non-zero one-form on each smooth fiber $E_{u}$. We choose the analytical marking that identifies the canonical differential $d x / y$ (where $(x, y)$ are the Weierstrass coordinates in Eq. (2.1) on the fiber $E_{u}$ ) with the holomorphic one-form $d z$. Given the elliptic surface $\mathrm{Z} \rightarrow \mathrm{UP}$ and the analytic marking we associate to it a holomorphic symplectic two-form [8], given by

$$
\lambda=d u \wedge \frac{d x}{y} .
$$

Using the two-form $\lambda$ the period integrals of the elliptic fiber $E_{u}$ over $u$ with periods $2 \omega, 2 \omega^{\prime}$ can be written as follows

$$
\int_{\alpha_{u}} \lambda=2 \omega d u, \quad \int_{\beta_{u}} \lambda=2 \omega^{\prime} d u .
$$

Then, there is a globally defined, real closed non-vanishing two-form form $\Omega$ on $\stackrel{\circ}{\mathrm{UP}}$

$$
\boldsymbol{\Omega}=\int_{E_{u}} \lambda \wedge \overline{\boldsymbol{\lambda}}=8 i \operatorname{Im} \tau|\boldsymbol{\omega}|^{2} d u \wedge d \bar{u} .
$$

The $\bar{\partial}$-operator along the fiber $E_{u}=\pi^{-1}(u)$ is the operator

$$
\bar{\partial}: \Omega^{0,0}\left(E_{u}\right) \rightarrow \Omega^{0,1}\left(E_{u}\right) .
$$

Its adjoint will be denoted by $\bar{\partial}^{*}$. We have the following lemma:

Lemma 4.1. The regularized determinant $\operatorname{det}^{\prime}\left(\bar{\partial}^{*} \bar{\partial}\right)$ of the Laplace operator along the fiber is a smooth function on UP given by

$$
\operatorname{det}^{\prime}\left(\bar{\partial}^{*} \bar{\partial}\right)=\operatorname{det}^{\prime} \Delta=\frac{\operatorname{vol}\left(E_{u}\right)^{2}}{(2 \pi)^{4}}\left|\Delta^{\frac{1}{12}}\right|^{2}
$$

where $\boldsymbol{\Delta}$ is the modular discriminant of the elliptic fiber $E_{u}$. 
Proof. It follows from Eq. (3.4) that

$$
\operatorname{det}^{\prime}\left(\bar{\partial}^{*} \bar{\partial}\right)=\operatorname{det}^{\prime} \Delta=\frac{\operatorname{vol}\left(E_{u}\right)^{2}}{(2 \pi)^{2}}\left|\frac{\eta^{2}(\tau)}{2 \boldsymbol{\omega}}\right|^{2} .
$$

The discriminant $\Delta$ of the elliptic curve $E_{u}$ is given by $\Delta=(2 \pi)^{12} \frac{\eta^{24}(\tau)}{(2 \boldsymbol{\omega})^{12}}$.

Finally, we have $\operatorname{vol}\left(E_{u}\right)=4 \operatorname{Im} \tau|\boldsymbol{\omega}|^{2}$.

We write $d \mathbf{a}=\boldsymbol{\omega} d u$ and $d \mathbf{a}_{D}=\boldsymbol{\omega}^{\prime} d u$ such that

$$
\tau=\frac{\boldsymbol{\omega}^{\prime}}{\boldsymbol{\omega}}=\frac{d \mathbf{a}_{D}}{d \mathbf{a}} .
$$

This notation should not suggest that $d \mathbf{a}$ is integrable, i.e., that there is a globally defined function a. On every open set $U \subset \mathrm{UP}$, we can integrate and find holomorphic functions $\left(\mathbf{a}, \mathbf{a}_{D}\right)$ such that on $U$ we have $\boldsymbol{\omega}=\frac{d \mathbf{a}}{d u}$ and $\boldsymbol{\omega}^{\prime}=$ $\frac{d \mathbf{a}_{D}}{d u}$. The Kähler metric (4.2) becomes $\boldsymbol{\Omega}=8 i \operatorname{Im} \tau d \mathbf{a} \wedge d \overline{\mathbf{a}}$. The following lemma was proved in [8]:

Lemma 4.2. The Levi-Civita connection $\nabla^{\mathrm{LC}}$ on $\stackrel{\mathrm{U} P}{\mathrm{P}}$ is given by

$$
\nabla^{\mathrm{LC}} \frac{\partial}{\partial \mathbf{a}}=-\frac{i}{2 \operatorname{Im} \tau} d \tau \otimes \frac{\partial}{\partial \mathbf{a}}, \quad \nabla^{\mathrm{LC}} \frac{\partial}{\partial \overline{\mathbf{a}}}=\frac{i}{2 \operatorname{Im} \tau} d \bar{\tau} \otimes \frac{\partial}{\partial \overline{\mathbf{a}}} .
$$

The scalar curvature of the Levi-Civita connection is

$$
S=\frac{1}{8 \operatorname{Im}^{3} \tau}\left|\frac{\partial \tau}{\partial \mathbf{a}}\right|^{2} .
$$

Proof. Let $\pi^{(1,0)} \in \Omega^{(1,0)}\left(T_{\mathbb{C}} \mathrm{UP}\right)$ be the projection onto the $(1,0)$ part of the complexified tangent bundle. $\pi^{(1,0)}$ is a one-form with values in the tangent bundle $T(\mathrm{UP})$. The Levi-Civita connection $\nabla^{\mathrm{LC}}$ on $\stackrel{\circ}{\mathrm{UP}}$ is defined by

$$
d\left[\boldsymbol{\Omega}\left(\frac{\partial}{\partial \mathbf{a}}, \frac{\partial}{\partial \overline{\mathbf{a}}}\right)\right]=\boldsymbol{\Omega}\left(\nabla^{\mathrm{LC}} \frac{\partial}{\partial \mathbf{a}}, \frac{\partial}{\partial \overline{\mathbf{a}}}\right)-\boldsymbol{\Omega}\left(\frac{\partial}{\partial \mathbf{a}}, \nabla^{\mathrm{LC}} \frac{\partial}{\partial \overline{\mathbf{a}}}\right) .
$$

It is the unique connection which is compatible with the metric and the complex structure. It follows that the Levi-Civita connection satisfies $\boldsymbol{\Omega}\left(\pi^{(1,0)}, \nabla^{\mathrm{LC}} \pi^{(1,0)}\right)=0$. Eqns. (4.5) follow. We find

$$
\begin{aligned}
d^{\nabla^{\mathrm{LC}}}\left(\nabla^{\mathrm{LC}} \frac{\partial}{\partial \mathbf{a}}\right) & =-\frac{i}{2} d\left(\frac{1}{\operatorname{Im} \tau}\right) \wedge d \tau \otimes \frac{\partial}{\partial \mathbf{a}}-\frac{i}{2 \operatorname{Im} \tau} d \tau \wedge \nabla^{\mathrm{LC}} \frac{\partial}{\partial \mathbf{a}} \\
& =-\frac{1}{4 \operatorname{Im}^{2} \tau} d \tau \wedge d \bar{\tau} \otimes \frac{\partial}{\partial \mathbf{a}}
\end{aligned}
$$

The Riemannian curvature $R$ of the Kähler metric $\boldsymbol{\Omega}$ is

$$
R=\left(\begin{array}{cc}
R_{a}^{a} a \bar{a} & 0 \\
0 & R_{\bar{a}}^{\bar{a}} a \bar{a}
\end{array}\right) d \mathbf{a} \wedge d \overline{\mathbf{a}}
$$


where

$$
R_{a{ }_{a \bar{a}}}^{a}=d \mathbf{a}\left\{d^{\nabla^{\mathrm{LC}}}\left(\nabla^{\mathrm{LC}} \frac{\partial}{\partial \mathbf{a}}\right)\left(\partial_{\mathbf{a}}, \partial_{\mathbf{a}}\right)\right\}=-\frac{1}{4 \operatorname{Im}^{2} \tau}\left|\frac{\partial \tau}{\partial \mathbf{a}}\right|^{2} .
$$

Pulling down the summation index using the metric $\Omega=i h_{a \bar{a}} d \mathbf{a} \wedge d \overline{\mathbf{a}}$ we find

$$
R_{a \bar{a} a \bar{a}}=h_{a \bar{a}} R_{a a \bar{a}}^{a}=-\frac{2}{\operatorname{Im} \tau}\left|\frac{\partial \tau}{\partial \mathbf{a}}\right|^{2} .
$$

The scalar curvature is obtained by contracting the summation indices using the inverse Kähler metric $\Omega$. We obtain

$$
S=-4\left(h^{a \bar{a}}\right)^{2} R_{a \bar{a} a \bar{a}}=\frac{1}{8 \operatorname{Im}^{3} \tau}\left|\frac{\partial \tau}{\partial \mathbf{a}}\right|^{2} .
$$

We obtain the following proposition:

Proposition 4.3. The function $F^{(1)}=-\frac{1}{2} \ln \operatorname{det}^{\prime} \Delta$ is a smooth function on UP and satisfies the equation

$$
\Delta_{\mathrm{UP}} F^{(1)}=S
$$

where $\Delta$ is the Laplace operator along the fiber of $\mathrm{Z} \rightarrow \mathrm{UP}, S$ is the scalar curvature of the Kähler metric $\boldsymbol{\Omega}$ on $\stackrel{\circ}{\mathrm{UP}}$, and $\Delta_{\mathrm{UP}}$ is the Laplace-Beltrami operator

$$
\Delta_{\mathrm{UP}}=\frac{1}{\operatorname{Im} \tau} \partial_{a} \partial_{\bar{a}}
$$

Proof. The proof follows from Lemma 4.1 and Lemma 4.2.

REMARK. Eq. (4.9) is the anomaly equation of the one-loop topological string amplitude derived in [4], i.e.,

$$
\partial_{a} \partial_{\bar{a}} F^{(1)}=\frac{1}{8 \operatorname{Im}^{2} \tau}\left|\frac{\partial \tau}{\partial \mathbf{a}}\right|^{2} .
$$

\section{The vertical $\bar{\partial}$-operator on $\mathrm{Z} \rightarrow \mathrm{UP}$}

In Section 3, we computed the regularized determinant of the $\bar{\partial}$-operator on an elliptic curve. When the elliptic curve $E_{u}$ is varied in an elliptic surface, we obtain the determinant line bundle DET $\bar{\partial} \rightarrow$ UP of the $\bar{\partial}$-operator along the fiber of $\mathrm{Z} \rightarrow \mathrm{UP}$. The determinant line bundle is the holomorphic line bundle

$$
\operatorname{DET} \bar{\partial} \rightarrow \stackrel{\circ}{\mathrm{UP}} \quad \text { with fibers } \quad(\operatorname{DET} \bar{\partial})_{u}=H^{0,0}\left(E_{u}, \mathbb{C}\right)^{-1} \otimes H^{0,1}\left(E_{u}, \mathbb{C}\right)
$$


There is a factorization of the determinant line bundle $\mathcal{L}=\mathrm{DET} \bar{\partial}$ as the tensor product of $\mathcal{L}^{\prime}$ and $\mathcal{H}$, corresponding to the non-zero and zero eigenvalues respectively. The line bundle $\mathrm{DET} \bar{\partial}$ can be identified with $\mathcal{H}$ since

$$
H^{0,0}\left(E_{u}, \mathbb{C}\right) \cong \operatorname{ker} \bar{\partial}, \quad H^{0,1}\left(E_{u}, \mathbb{C}\right) \cong \operatorname{ker} \bar{\partial}^{*} .
$$

The bundle $\mathcal{L}^{\prime}$ has a holomorphic section $\operatorname{det}^{\prime} 2 \bar{\partial}$ that determines an isomorphism DET $\bar{\partial} \cong \mathcal{H}[\mathbf{2}]$. The isomorphism does not preserve the metric or connection. Bismut and Freed defined the smooth metric

$$
\|\cdot\|_{Q}:=(2 \pi)^{2} \sqrt{\operatorname{det}^{\prime} \Delta}\|\cdot\|_{L^{2}}
$$

on DET $\bar{\partial}$ and determined its unitary connection. Since $\omega_{Z / \mathrm{UP}}$ is equipped with a Hermitian $C^{\infty}$-metric, the Quillen metric is a Hermitian $C^{\infty}$-metric on the holomorphic fibers $(\mathrm{DET} \bar{\partial})_{u}$. The curvature of this connection is called the local anomaly in physics. It follows:

LEMma 5.1. The determinant line bundle $\mathrm{DET} \bar{\partial} \rightarrow \stackrel{\circ}{\mathrm{UP}}$ is flat. $\sigma=(d z)^{-1}$ is a non-vanishing holomorphic section of DET $\bar{\partial}$ with

$$
\|\sigma\|_{Q}=|\Delta|^{\frac{1}{12}} .
$$

$\sigma^{*}=d z$ is a non-vanishing holomorphic section of the dual bundle $(\operatorname{DET} \bar{\partial})^{*} \rightarrow \stackrel{\circ}{\mathrm{UP}}$ with $\left\|\sigma^{*}\right\|_{Q^{*}}=|\boldsymbol{\Delta}|^{-\frac{1}{12}}$.

Proof. The flatness follows from the curvature formula of Bismut and Freed ([7] or [2, Prop. 5.14]):

$$
c_{1}(\operatorname{DET} \bar{\partial})=-\int_{E_{u}} \frac{c_{1}^{2}\left(\omega_{\mathrm{Z} / \mathrm{UP}}\right)}{6} .
$$

It follows from Remark 2 that $c_{1}^{2}\left(\omega_{\mathrm{Z} / \mathrm{UP}}\right)=0$.

$H^{0,1}\left(E_{u}\right)$ and $H^{1,0}\left(E_{u}\right)$ are Serre duals. Thus, we have $\mathcal{H} \cong\left[H^{1,0}\left(E_{u}\right) \otimes\right.$ $\left.H^{0,0}\left(E_{u}\right)\right]^{-1}$. The kernel consists of the constant function $\phi=1$ with $\|\phi\|^{2}=$ $\operatorname{vol}\left(E_{u}\right)$. By Serre duality we identify the cokernel ker $\bar{\partial}^{*}$ with the dual of the space of holomorphic one-forms. Thus, the cokernel is spanned by the section $(d z)^{-1}$ and $\|d z\|^{2}=\operatorname{vol}\left(E_{u}\right)$. Using Eq. (3.4) we obtain for the Quillen norm of the section $(d z)^{-1}$

$$
\left\|(d z)^{-1}\right\|_{Q}^{2}=(2 \pi)^{4} \frac{\operatorname{det}(-4 \partial \bar{\partial})_{(1,1)}}{\|\phi\|^{2}\|d z\|^{2}}=|\Delta|^{\frac{2}{12}} .
$$

It is possible to factorize the right hand side holomorphically in $\tau$. We use the Quillen metric to obtain a smooth section $\sigma^{\#}$ of the dual bundle (DET $\left.\bar{\partial}\right) *$ by setting

$$
\sigma^{\#}=g_{Q}(\sigma, \bullet)=|\Delta|^{\frac{1}{6}} d z,
$$

and $\left\|\sigma^{\#}\right\|_{Q^{*}}=|\boldsymbol{\Delta}|^{1 / 12}$ by definition. The claim follows. 
REMARK. Under the change of the coordinate chart from $[u: 1]$ to $[1: v]$ on $\mathbb{C P}^{1}$ by $u=-1 / v$ the holomorphic differential transforms as $d z_{u}=\frac{d x_{u}}{y_{u}}=$ $-v \frac{d x_{v}}{y_{v}}=-v d z_{v}$. The Quillen metric is compatible with a change of coordinates since

$$
\left\|\left(d z_{v}\right)^{-1}\right\|_{Q}^{2}=|v|^{2}\left\|\left(d z_{u}\right)^{-1}\right\|_{Q}^{2}=|v|^{2}\left|\Delta_{u}^{\frac{1}{12}}\right|^{2}=\left|\Delta_{v}^{\frac{1}{12}}\right|^{2} .
$$

Lemma 5.2. The Bismut-Freed connection on $\mathrm{DET} \bar{\partial} \rightarrow \stackrel{\circ}{\mathrm{UP}}$ is flat and given by

$$
\nabla^{\mathrm{BF}} \sigma=\frac{1}{12} \frac{\partial \Delta}{\Delta} \otimes \sigma
$$

and $\nabla^{\mathrm{BF}(0,1)}=\bar{\partial}$. $\mathbb{C P}^{1}$

Proof. It follows for the Quillen metric in the coordinate chart $[u: 1] \in$

$$
\frac{d\|\sigma\|_{Q}^{2}}{\|\sigma\|_{Q}^{2}}=\frac{1}{12} \frac{\partial \ln \Delta}{\partial u} d u+\frac{1}{12} \frac{\partial \ln \bar{\Delta}}{\partial \bar{u}} d \bar{u}
$$

Although DET $\bar{\partial}$ is flat and hence the local anomaly vanishes, there is a global anomaly arising from monodromy around the non-contractible closed loops. These are the non-contractible loops $\left(\gamma_{n}\right)_{n=1}^{N_{f}+2}$ encircling the nodes at $\left(u_{n}^{*}\right)_{n=1}^{N_{f}+2}$ clockwise, and $\gamma_{\infty}$ encircling the cusp at infinity counterclockwise.

Lemma 5.3. There exist constants in $\mathbb{R}^{+}$such that

$$
\begin{array}{rlrl}
\|\sigma\|_{Q} & \sim c_{n}\left|u-u_{n}^{*}\right|^{\frac{1}{12}} & \left(u \rightarrow u_{n}^{*}\right), \\
\|\sigma\|_{Q} \sim c_{\infty}|v|^{\frac{10-N_{f}}{12}} & (v \rightarrow 0) .
\end{array}
$$

Proof. The proof follows from Lemma 5.1, Remark 5, and the fact that $\Delta$ has a simple zero at each node $u_{n}$ and is a polynomial of degree $N_{f}+2$ in $u$.

We denote the holonomy of the determinant section of DET $\bar{\partial}$ on the boundary circle $\gamma_{n}$ around $u_{n}$ by $\exp \left(-\frac{i \pi}{2} \eta_{\bar{\partial}}\left[\gamma_{n}\right]\right)$. The following lemma is an immediate consequence of Lemma 5.3:

LEMma 5.4. The holonomy of the section $\sigma$ on a cycle $\gamma_{i}$ is $\exp \left(-\frac{\pi i}{2}\right.$ $\left.\eta_{\bar{\partial}}\left[\gamma_{i}\right]\right)$ with

$$
\eta_{\bar{\partial}}\left[\gamma_{n}\right] \equiv-\frac{1}{3} \quad \bmod 4, \quad \eta_{\bar{\partial}}\left[\gamma_{\infty}\right] \equiv-\frac{10-N_{f}}{3} \quad \bmod 4
$$


Since we have restricted ourselves to the case where the fibration $\mathrm{Z} \rightarrow \mathrm{UP}$ has no surface singularities, the singular fibers $E_{u}=\pi^{-1}(u)$ at $u=u_{1}^{*}, \ldots$, $u_{N_{f}+2}^{*}$ are nodal curves. A current $\delta\left(u-u_{n}^{*}\right)$ on $\mathrm{Z} \rightarrow \mathrm{UP}$ is defined by saying that for every differential form $\alpha$ on $\mathrm{Z}$ with compact support, the equality

$$
\int_{Z} \delta\left(u-u_{n}^{*}\right) d u \wedge d \bar{u} \wedge \alpha=\int_{E_{u_{n}^{*}}} \alpha
$$

holds. The first Chern class $c_{1}\left(\operatorname{DET} \bar{\partial},\|\cdot\|_{Q}\right)$ is defined as a current of type $(1,1)$ by

$$
c_{1}\left(\operatorname{DET} \bar{\partial},\|\cdot\|_{Q}\right):=\frac{1}{2 \pi i} \partial \bar{\partial} \log \|\sigma\|_{Q}^{2}
$$

where $\sigma$ is a local non-vanishing holomorphic section.

Proposition 5.5. The determinant line bundle with the Quillen metric $\|.\|_{Q}$ of the $\bar{\partial}$-operator along the fiber of $\mathrm{Z} \rightarrow \mathrm{UP}$ extends to a holomorphic line bundle DET $\bar{\partial} \rightarrow \mathrm{UP}$ with curvature

$$
c_{1}(\operatorname{DET} \bar{\partial})=-\sum_{n=1}^{N_{f}+2} \frac{1}{12} \delta\left(u-u_{n}^{*}\right) d u \wedge d \bar{u} .
$$

Proof. The definition of the holomorphic determinant line bundle in Eq. (5.1) can be extended across the singular fibers of an elliptic fibration using the results of Knudsen and Mumford [5]. The Quillen metric is smooth by Lemma 4.4. Let $f$ be a function that is differentiable in the disc $D_{\epsilon}$ with $|u|<\epsilon$. Suppose further that $f$ and its derivatives with respect to $\bar{u}$ are bounded on the disc. Let $\mathcal{T}$ denote the function

$$
(\mathcal{T} f)(u, \bar{u})=\frac{1}{2 \pi i} \iint_{|w|<\epsilon} f(w, \bar{w}) \frac{d w d \bar{w}}{u-w}
$$

It is well-known [15] that the linear operator $\mathcal{T}$ is differentiable and admissible on $D_{\epsilon}$ and satisfies $\bar{\partial}(\mathcal{T} f)=f$. In this sense, we write

$$
\frac{1}{2 \pi i} \frac{\partial}{\partial \bar{u}} \frac{1}{u-w}=\delta(u-w) .
$$

Since $\bar{\partial} \partial+\partial \bar{\partial}=0$, Eq. (5.4) follows from Eq. (5.3) and the application of Lemma 5.3.

REMARK. Corollary 5.5 shows that we can include the nodal fibers of $\mathrm{Z} \rightarrow \mathrm{UP}$ when considering the determinant line bundle of the $\bar{\partial}$-operator along the fiber. The contributions $\eta_{\bar{\partial}}\left[\gamma_{n}\right]$ to the global anomaly of the determinant line bundle around the nodal fibers are then viewed as current contributions of type $(1,1)$ to the first Chern class of the extended determinant line bundle. 
The results in $[\mathbf{5}$, Thm. 2.1] and $[\mathbf{6}]$ describe the more general situation of the $\bar{\partial}$-operator coupled to a holomorphic vector bundle $V \rightarrow \mathrm{Z}$. There, the authors prove that

$$
\begin{aligned}
c_{1}\left(\operatorname{DET} \bar{\partial}_{V},\|\cdot\|_{Q}\right)= & -\int_{\pi}\left[\operatorname{ch}(V) \wedge \operatorname{Todd}\left(\omega_{Z / \mathrm{UP}},\|\cdot\|\right)\right]_{(4)} \\
& -\sum_{n} \frac{\operatorname{rank}(V)}{12} \delta\left(u-u_{n}^{*}\right) d u \wedge d \bar{u} .
\end{aligned}
$$

For $\operatorname{Todd}\left(\omega_{Z / \mathrm{UP}},\|\|.\right)=1+c_{1}\left(\omega_{Z / \mathrm{UP}}\right) / 2\left(\operatorname{since} c_{1}^{2}\left(\omega_{Z / \mathrm{UP}}\right)=0\right)$, and $\operatorname{ch}(V)=$ 1 we obtain Eq. (5.4).

\section{Extending $\bar{\partial}$ to nodal curves}

Let us restrict the fibration $\mathrm{Z} \rightarrow \mathrm{UP}$ to a small neighborhood $U$ of a point $u^{*}$ whose singular fiber is a nodal curve. We identify any smooth elliptic fiber $E_{u}$ of the fibration $\mathrm{Z} \rightarrow U$ with the complex plane (with complex coordinate $z$ ) modulo the action of the lattice generated by the periods $2 \omega, 2 \omega^{\prime}$. For $\tau=\frac{\omega^{\prime}}{\omega}$ we set $q=\exp 2 \pi i \tau$. After a suitable $\operatorname{SL}(2, \mathbb{Z})$ transformation we can assume that as we approach the node for $u \rightarrow u^{*}$ we have $\operatorname{Im} \tau \rightarrow \infty, q \rightarrow 0$. By making the neighborhood $U$ smaller if necessary, we assume that $|q|<1$ uniformly in $U$.

Next, we consider the annulus $\operatorname{ann}\left(r_{1}, r_{2}\right)$ in $\mathbb{C}$ (with complex coordinate $W$ ) with inner radius $0<r_{1}<1$ and outer radius $r_{2}=1 / r_{1}$. We also set $Z=1 / W$ for $W \neq 0$. If we set $r_{1}=|q|^{1 / 2}$, then the annulus is covered by the two charts $r_{1}<|W| \leq 1$ and $r_{1}<|Z| \leq 1$. The inner and outer radius of the annulus are identified to obtain a torus using $Z W=q$. This is the local description of a compact Riemann surface near a node used in [18] when applied to a torus. For $q \rightarrow 0$ we get a singular surface envisioned as sphere with two points $\{Z=0\}$ and $\{W=0\}$ identified.

The map $W=r_{2} \exp \left(2 \pi i \frac{z}{2 \omega}\right)$ identifies the fundamental domain for the torus $E_{u} \cong \mathbb{C} /\left\langle 2 \omega, 2 \omega^{\prime}\right\rangle$ with its vertical edges parallel to $2 \omega^{\prime}$ identified with the annulus $\operatorname{ann}\left(r_{1}, r_{2}\right)$. With respect to the metric $g=d z . d \bar{z}$ we have $\operatorname{vol}\left(E_{u}\right)=4 \operatorname{Im} \tau|\boldsymbol{\omega}|^{2}$ and

$$
g=d z \cdot d \bar{z}=\left(\frac{|\boldsymbol{\omega}|}{\pi|W|}\right)^{2} d W \cdot d \bar{W}
$$

The following lemma computes the regularized determinant for the LaplaceBeltrami operator on the annulus with respect to the metric (6.1) and with Dirichlet boundary conditions, i.e., for eigenfunctions vanishing on the outer and inner radius:

Lemma 6.1. The regularized determinant for the Laplace operator $\Delta=$ $-4 \partial_{z} \bar{\partial}_{z}$ on the annulus $\operatorname{ann}\left(r_{1}, r_{2}\right)$ with Dirichlet boundary conditions is 
given by

$$
\operatorname{det}_{D} \Delta=\frac{\operatorname{Im} \tau}{2 \pi}|\boldsymbol{\eta}(\tau)|^{2}
$$

Proof. The eigenfunctions $\psi_{n_{1}, n_{2}}\left(\xi^{1}, \xi^{2}\right)$ of the Laplace-Beltrami operator with Dirichlet boundary conditions are similar to the eigenfunctions in section 3 . In the case $\left(\nu_{1}, \nu_{2}\right)=(1,1)$ we have

$$
\begin{aligned}
\varphi_{n_{1}, n_{2}}\left(\xi^{1}, \xi^{2}\right)= & \exp \left[2 \pi i n_{1}\left(\xi^{1}-\frac{\operatorname{Re} \tau}{\operatorname{Im} \tau} \xi^{2}\right)\right]\left[\cos \left(\frac{2 \pi n_{2}}{\operatorname{Im} \tau} \xi^{2}\right)\right. \\
& \left.+i \sin \left(\frac{2 \pi n_{2}}{\operatorname{Im} \tau} \xi^{2}\right)\right] .
\end{aligned}
$$

The Dirichlet boundary conditions are $\psi_{n_{1}, n_{2}}\left(\xi^{1}, 0\right)=\psi_{n_{1}, n_{2}}\left(\xi^{1}, \operatorname{Im} \tau\right)=0$. Hence, we have $n_{2}>0$ and

$$
\psi_{n_{1}, n_{2}}\left(\xi^{1}, \xi^{2}\right)=\exp \left[2 \pi i n_{1}\left(\xi^{1}-\frac{\operatorname{Re} \tau}{\operatorname{Im} \tau} \xi^{2}\right)\right] \sin \left(\frac{2 \pi n_{2}}{\operatorname{Im} \tau} \xi^{2}\right) .
$$

Therefore, the zeta-function $\zeta_{D}(s)$ for the Laplace operator with Dirichlet boundary conditions is

$$
\zeta_{D}(s)=\sum_{n_{1}, n_{2}>0} \frac{1}{\left[n_{1}^{2} \operatorname{Im}^{2} \tau+\left(n_{1} \operatorname{Re} \tau-n_{2}\right)^{2}\right]^{s}}=\frac{1}{2}\left(\zeta_{(1,1)}(s)-\frac{2}{|\tau|^{2 s}} \zeta(2 s)\right),
$$

where $\zeta_{(1,1)}(s)$ was defined in Eq. (3.3) and $\zeta(2 s)=\sum_{n=1}^{\infty} n^{-2 s}$ is the Riemann zeta function. Thus, we have

$\zeta_{D}(0)=\frac{1}{2}\left(\zeta_{(1,1)}(0)-2 \zeta(0)\right), \quad \zeta_{D}^{\prime}(0)=\frac{1}{2}\left(\zeta_{(1,1)}^{\prime}(0)+4 \ln |\tau| \zeta(0)-4 \zeta^{\prime}(0)\right)$.

Eq. (6.2) then follows from $\zeta(0)=-1 / 2, \zeta^{\prime}(0)=-\ln (2 \pi) / 2$, and Eq. (3.4).

Instead of the Laplacian $\Delta=-4 \partial_{z} \bar{\partial}_{z}$ Seeley and Singer [18] use the Laplace-Beltrami operator for the flat metric on the annulus. The flat metric $\widehat{g}$ on the annulus is obtained from the metric $g$ in Eq. (6.1) by a change in the conformal gauge, i.e.,

$$
\widehat{g}=d W \cdot d \bar{W}=e^{2 \Phi} g
$$

with $\Phi=\ln (\pi|W| /|\boldsymbol{\omega}|)$. It follows that the Laplace-Beltrami operator for the flat metric is given by

$$
\widehat{\Delta}=-4 \partial_{W} \bar{\partial}_{W}=e^{-2 \Phi} \Delta .
$$

We use the results of $[\mathbf{2 1}]$ to derive the following lemma: 
LEMmA 6.2. The regularized determinant of the Laplace operator $\widehat{\Delta}$ on the annulus $\operatorname{ann}\left(r_{1}, r_{2}\right)$ with Dirichlet boundary conditions is given by

$$
\operatorname{det}_{D} \widehat{\Delta}=\frac{\operatorname{Im} \tau}{2 \pi}|\boldsymbol{\eta}(\tau)|^{2}|q|^{\frac{1}{6}} .
$$

Proof. The Gaussian curvature as well as the geodesic curvature on the boundary vanish for $g$. Similarly, the Gaussian curvature and the average geodesic curvature on the boundary vanish for $\widehat{g}$. Then, $[\mathbf{2 1}$, Eq. (3)] implies

$$
\operatorname{det}_{D} \Delta=\operatorname{det}_{D} \widehat{\Delta} \exp \left(\frac{L}{6 \pi}\right)
$$

where $L=\frac{1}{2} \int_{\operatorname{ann}\left(r_{1}, r_{2}\right)} \operatorname{vol}_{g} g^{a b}\left(\partial_{a} \Phi\right)\left(\partial_{b} \Phi\right)$. A calculation shows that

$$
L=\frac{i}{4} \int_{\operatorname{ann}\left(r_{1}, r_{2}\right)} \frac{d W \wedge d \bar{W}}{|W|^{2}}=\pi \ln \left(\frac{r_{2}}{r_{1}}\right)=2 \pi^{2} \operatorname{Im} \tau
$$

and $\exp L / 6 \pi=|q|^{(-1 / 6)}$. We obtain

$$
\operatorname{det}_{D} \Delta=\operatorname{det}_{D} \widehat{\Delta}|q|^{-\frac{1}{6}} \text {. }
$$

The application of the result of Seeley and Singer $[\mathbf{1 8}]$ yields the following proposition:

Proposition 6.3. In a small neighborhood $U \subset \mathrm{UP}$ of a point $u^{*}$ with nodal fiber $E_{u^{*}}$ such that $q=\exp (2 \pi i \tau) \rightarrow 0$ as $u \rightarrow u^{*}$ the family of operators $\left\{\bar{\partial}_{W, u}\right\}_{u \in U}$ is a continuous family and the operator $\bar{\partial}_{W}$ is well-defined on the singular fiber $E_{u^{*}}$.

REMARK. The limiting Laplace operator of $[\mathbf{1 8}]$ is the Laplace operator $-4 \partial_{W} \bar{\partial}_{W}$ on $\mathbb{C}$ (with complex coordinate $W$ ). The eigenfunction for an eigenvalue $\lambda^{2}$ with $\lambda>0$ satisfying Dirichlet boundary conditions is $\mathrm{J}_{n}(\lambda r)$ $\exp (i n \theta)$ with $W=r \exp (i \theta)$ and $n \in \mathbb{N}$. $\mathrm{J}_{n}(\lambda r)$ is the Bessel function of the first kind that is regular at $r=0$ and decays as $1 / \sqrt{r}$ for $r \rightarrow \infty$.

\section{The vertical signature operator on $\mathrm{Z} \rightarrow \mathrm{UP}$}

Using its complex structure the signature operator on each fiber $E_{u}$ can be identified with the operator

$$
\mathrm{D}=\bar{\partial}+\bar{\partial}_{1}: \quad \Omega^{0,0}\left(E_{u}\right) \oplus \Omega^{1,0}\left(E_{u}\right) \rightarrow \Omega^{0,1}\left(E_{u}\right) \oplus \Omega^{1,1}\left(E_{u}\right) .
$$

Again, there is a factorization of the determinant line bundle $\mathcal{L}=\mathrm{DET} \mathrm{D}$ as the tensor product of $\mathcal{L}^{\prime}$ and $\mathcal{H}$, corresponding to the non-zero and zero eigenvalues respectively [2]. The bundle $\mathcal{L}^{\prime}$ has the holomorphic section $\operatorname{det}^{\prime} \mathrm{D}$. The fiber of the line bundle $\mathcal{H}$ is

$$
\mathcal{H} \cong\left[H^{0,0}\left(E_{u}\right) \otimes H^{1,0}\left(E_{u}\right)\right]^{-1} \otimes\left[H^{0,1}\left(E_{u}\right) \otimes H^{1,1}\left(E_{u}\right)\right] .
$$


The bundles $H^{0,0}\left(E_{u}\right)$ and $H^{1,1}\left(E_{u}\right)$ can be identified by duality. Similarly, $H^{0,1}\left(E_{u}\right)$ and $H^{1,0}\left(E_{u}\right)$ are Serre duals. On each fiber $E_{u}$ multiplication by $d z$ converts $\bar{\partial}$ into $\bar{\partial}_{1}$. Thus, we have $\mathcal{H} \cong\left[H^{1,0}\left(E_{u}\right)\right]^{-2}$ and the determinant line bundle of the operator $\mathrm{D}$ is isomorphic to the two-fold tensor product of DET $\bar{\partial}$.

We have the following lemma:

LEMMA 7.1.

(1) $\sigma_{\mathrm{D}}=(d z)^{-2}$ is a non-vanishing holomorphic section of DET $\mathrm{D} \rightarrow$ UP with

$$
\begin{aligned}
& \qquad \sigma_{\mathrm{D}} \|_{Q}=|\Delta|^{\frac{1}{6}} \text {. } \\
& \sigma_{\mathrm{D}}^{*}=(d z)^{2} \text { is a holomorphic section of the dual bundle }(\mathrm{DET} \mathrm{D})^{*} \rightarrow \\
& \text { UP with }\left\|\sigma_{\mathrm{D}}^{*}\right\|_{Q^{*}}=|\Delta|^{-\frac{1}{6}} \text {. }
\end{aligned}
$$

(2) The flat Bismut-Freed connection on $\mathrm{DET} \mathrm{D} \rightarrow \stackrel{\mathrm{U} P}{\mathrm{UP}}$ is given by

$$
\nabla^{\mathrm{BF}} \sigma_{\mathrm{D}}=\frac{1}{6} \frac{\partial \Delta}{\Delta} \otimes \sigma_{\mathrm{D}}
$$

and $\nabla^{\mathrm{BF}(0,1)}=\bar{\partial}$.

(3) The determinant line bundle with the Quillen metric extends to a holomorphic line bundle $\mathrm{DET} \mathrm{D} \rightarrow \mathrm{UP}$. The curvature is a current with

$$
c_{1}(\mathrm{DET} \mathrm{D})=-\sum_{n=1}^{N_{f}+2} \frac{1}{6} \delta\left(u-u_{n}^{*}\right) d u \wedge d \bar{u} .
$$

Proof. The proof is the same as for Lemma 5.2.

Lemma 7.2. The line bundle (DET D)*6 $\rightarrow \stackrel{\circ}{\mathrm{UP}}$ is canonically trivial.

Proof. The section $\underline{\sigma}=\Delta^{1 / 6}(d z)^{2} \quad$ of $\quad(\mathrm{DET} \mathrm{D})^{*} \rightarrow \stackrel{\circ}{\mathrm{UP}}$ satisfies $\|\underline{\sigma}\|_{Q^{*}}=1$ and is invariant under the action of $\pi_{1}(\mathrm{U} \mathrm{U})$ up to a sixth root of unity. The trivializing, holomorphic, non-vanishing section of (DET $\mathrm{D})^{* \otimes 6} \rightarrow \stackrel{\circ}{\mathrm{UP}}$ is $\underline{\sigma}^{6}$.

It follows from Remark 1 that $\mathcal{H} \cong T^{*} \mathrm{UP}$. Consequently, we can obtain well-defined logarithmic monodromies for the bundle (DET D) $\rightarrow \stackrel{\mathrm{UP}}{\mathrm{U}}$. We denote this distinguished choice for the monodromy by $\eta_{\mathrm{D}}^{0}[\gamma]$ as opposed to $\eta_{\mathrm{D}}[\gamma]$ which appeared in Lemma 5.4 and was only determined modulo 4.

LEMma 7.3. The logarithmic monodromies of the bundle (DET D)* $\rightarrow$ UP are

$$
\eta_{\mathrm{D}}^{0}\left[\gamma_{n}\right]=-\frac{2}{3}, \quad \eta_{\mathrm{D}}^{0}\left[\gamma_{\infty}\right]=-\frac{2\left(10-N_{f}\right)}{3}
$$


Proof. Under the isomorphism $\mathcal{H} \cong T^{*} \mathrm{UP}$ the form $(d z)^{2}$ is identified with $d u^{-1}$. Thus, the bundle $(\mathrm{DET} \mathrm{D})^{*} \otimes T^{*} \mathrm{UP} \rightarrow \stackrel{\circ}{\mathrm{UP}}$ has a standard trivialization on UP given by $(d z)^{2} \otimes d u^{-1}$. In this trivialization the holomorphic section is $\Delta^{1 / 6}$. The claim follows.

LEMMA 7.4. The signature of the elliptic surface $\mathrm{Z} \rightarrow \mathrm{UP}$ is

$$
\operatorname{sign}(\mathrm{Z})=\sum_{n=1}^{N_{f}+2} \eta_{\mathrm{D}}^{0}\left[\gamma_{n}\right]-\frac{1}{2} \eta_{\mathrm{D}}^{0}\left[\gamma_{\infty}\right]+2
$$

It follows $\operatorname{sign}(\mathrm{Z})=-N_{f}$.

Proof. The signature of the rational elliptic surface $\bar{Z} \rightarrow \mathbb{C P}^{1}$ in terms of its Chern classes $c_{1}, c_{2}$ is

$$
\operatorname{sign}(\overline{\mathrm{Z}})=\frac{c_{1}^{2}-2 c_{2}}{3} .
$$

The canonical class is minus the fiber class so that $c_{1}^{2}=0$ while $c_{2}$ is the sum of the exceptional fibers of the fibration whence

$$
\operatorname{sign}(\overline{\mathrm{Z}})=-\frac{2}{3} \sum_{n=1}^{N_{f}+2} e\left(E_{u_{n}^{*}}\right)-\frac{2}{3} e\left(E_{\infty}\right)
$$

The elliptic surface $\stackrel{\circ}{\mathrm{Z}} \rightarrow \stackrel{\circ}{\mathrm{UP}}$ is obtained by cutting out all singular fibers $\stackrel{\circ}{\mathrm{Z}}=\overline{\mathrm{Z}}-\cup E_{u_{n}^{*}}-E_{\infty}$; the elliptic surface $\mathrm{Z} \rightarrow \mathrm{UP}$ is obtained by cutting out only the singular fiber at infinity whence $\mathrm{Z}=\overline{\mathrm{Z}}-E_{\infty}$. Since the singular fibers at $u=u_{1}^{*}, \ldots, u_{N_{f}+2}^{*}$ are nodes they do not contribute to the signature. Hence, we have

$$
\operatorname{sign}(\stackrel{\circ}{Z})=\operatorname{sign}(Z)=\operatorname{sign}(\bar{Z})-\operatorname{sign}\left(E_{\infty}\right),
$$

where $E_{\infty}$ is the singular fiber of $\bar{Z}$ at infinity. Thus, we obtain

$$
\operatorname{sign}(\mathrm{Z})=-\frac{2}{3} \sum_{n=1}^{N_{f}+2} e\left(E_{u_{n}^{*}}\right)-\frac{2}{3} e\left(E_{\infty}\right)-\operatorname{sign}\left(E_{\infty}\right)
$$

The Euler number $e\left(E_{u_{n}^{*}}\right)$ is equal to the degree of the zero the discriminant assumes at $u=u_{n}^{*}$. Therefore, it follows $-\frac{2}{3} e\left(E_{u_{n}^{*}}\right)=\eta_{D}^{0}\left[\gamma_{n}\right]$ and $-\frac{2}{3} e\left(E_{\infty}\right)=$ $\eta_{D}^{0}\left[\gamma_{\infty}\right]$. By Kodaira's classification result it follows that singularities which are not of type $I_{k}$ satisfy $\operatorname{sign}\left(E_{\infty}\right)=2-e\left(E_{\infty}\right)$. Lemma 7.3 yields $\operatorname{sign}(\mathrm{Z})=$ $-N_{f}$. 
7.1. Regular singularities and the Riemann-Hilbert problem. The definition of the holomorphic determinant line bundle in Eq. (5.1) can be extended across the singular fibers of an elliptic fibration using the results of Knudsen and Mumford [5] to include the higher-rank singularities of Kodaira type $I_{k}$ and $I_{k}^{*}$. In this section, we allow any Jacobian rational elliptic surface $\mathrm{Z}$ with singular fibers of Kodaira type $I_{k_{n}}$ over $\left[u_{n}^{*}: 1\right] \in \mathbb{C P}^{1}$ (with $1 \leq n \leq K$ such that $\left.\sum k_{n}=N_{f}+2\right)$ and a singular fiber of Kodaira type $I_{4-N_{f}}^{*}$ over $u=\infty$. The following solution to the Riemann-Hilbert problem on $\mathbb{C P}^{1}$ was given by Röhrl in terms of differential equations with regular singular points $[\mathbf{1 0}]$ :

FACT 7.5.

(1) The functor mapping all conjugate classes of one-dimensional representations of $\pi_{1}(\mathrm{UP})$ to the set of isomorphism classes of flat line bundles over $\stackrel{\circ}{\mathrm{UP}}$ is an equivalence of categories.

(2) For a flat line bundle $\mathfrak{E} \rightarrow \stackrel{\circ}{\mathrm{UP}}$ together with the natural connection $d$ on $\stackrel{\circ}{\mathrm{UP}}$, there exists a holomorphic line bundle $\mathfrak{L} \rightarrow \mathbb{C P}^{1}$ together with an integrable connection $\nabla$ with regular singular points $u_{1}^{*}$, $u_{2}^{*}, \ldots$, such that the restriction of $\mathfrak{L}$ is an isomorphism $i: \mathfrak{L} \rightarrow \mathfrak{E}$ with

$$
\left.d \circ i\right|_{\mathrm{UP}} ^{\circ}=\left.(i \otimes 1) \circ \nabla\right|_{\mathrm{UP}} ^{\circ} .
$$

(3) The holomorphic line bundle $\mathfrak{L}$ admits a global meromorphic section $\sigma$, so $\mathfrak{L}$ is meromorphically trivial and the connection $\nabla$ coincides with a homomorphism defined by a global meromorphic Pfaffian system $(d-\theta) \rho=0$ where $\theta$ is a global meromorphic one-form on $\mathbb{C P}^{1}$. Since $\theta=d \rho / \rho$ the curvature $d \theta$ vanishes. Then, $d \theta$ can be prolonged to the whole $\mathbb{C P}^{1}$.

This implies the following result:

Proposition 7.6. On the holomorphic anti-canonical line bundle $\omega_{\mathbb{C P}^{1}}^{-1}$, there exists an integrable meromorphic connection $\nabla$ with regular singular points $u_{1}^{*}, u_{2}^{*}, \ldots, \infty$ such that the restriction of $\omega_{\mathbb{C P}^{1}}^{-1}$ to $\stackrel{\circ}{\mathrm{UP}}$ is isomorphic to the determinant line bundle $(\mathrm{DET} \mathrm{D})^{*} \rightarrow \stackrel{\circ}{\mathrm{UP}}$ of the signature operator along the fiber of $\mathrm{Z} \rightarrow \stackrel{\circ}{\mathrm{UP}}$. The curvature $\Omega$ of $\nabla$ equals

$$
\frac{i \Omega}{2 \pi}=\sum_{n} \frac{k_{n}}{6} \delta\left(u-u_{n}^{*}\right) d u \wedge d \bar{u}+\frac{10-N_{f}}{6} \delta(v) d v \wedge d \bar{v}
$$

whence $\int_{\mathbb{C P}^{1}} i \Omega /(2 \pi)=2$. 
Proof. The determinant line bundle $(\mathrm{DET} D)^{*} \rightarrow \stackrel{\circ}{\mathrm{UP}}$ of the signature operator along the fiber takes the place of the holomorphic flat line bundle $\mathfrak{E}$ in Fact 7.5. The bundle $\mathfrak{E}$ has the meromorphic section $\underline{\sigma}=\Delta^{1 / 6}(d z)^{2}$ with $\|\underline{\sigma}\|_{Q^{*}}=1$. Hence, the Bismut-Freed connection acts on $\underline{\sigma}$ simply as the exterior derivative $d$. Outside the set of singular points, the determinant line bundle is isomorphic to $\mathfrak{L}=\omega_{\mathbb{C P}^{1}}^{-1}$. In the chart $[u: 1] \in \mathbb{C P}^{1}$, the isomorphism is given by multiplication with $\rho_{u}=\Delta_{u}^{1 / 6}$ and identifying $d u^{-1}$ with $\left(d z_{u}\right)^{2}$ such that $i\left(d u^{-1}\right)=\rho_{u}(d z)^{2}$. The bundle $\mathfrak{L}$ carries the integrable meromorphic connection

$$
\nabla d u^{-1}=-\frac{d \rho_{u}}{\rho_{u}} \otimes d u^{-1}
$$

with regular singular points $u_{n}^{*}$. In particular, the form $\theta_{u}=d \rho_{u} / \rho_{u}$ has simple poles at every regular singular point $u_{n}^{*}$ and the counterclockwise contour integral evaluates to

$$
\frac{1}{2 \pi i} \oint_{u_{n}^{*}} \frac{d \rho_{u}}{\rho_{u}}=\frac{k_{n}}{6}
$$

Under a change of the coordinate chart from $[u: 1]$ to $[1: v]$ on $\mathbb{C P}^{1}$ by $u=-1 / v$ the holomorphic differential transforms as $d z_{u}=-v d z_{v}$. The isomorphism is given by multiplication with $\rho_{v}=\Delta_{v}^{1 / 6}=v^{2} \rho_{u}$ and identifying $d v^{-1}$ with $\left(d z_{v}\right)^{2}$ such that $i\left(d v^{-1}\right)=\rho_{v}\left(d z_{v}\right)^{2}$. In particular, the form $\theta_{v}=d \rho_{v} / \rho_{v}$ has simple pole at $v=0$ and the counterclockwise contour integral evaluates to

$$
\frac{1}{2 \pi i} \oint_{v=0} \frac{d \rho_{v}}{\rho_{v}}=\frac{10-N_{f}}{6}
$$

The connection one-forms $\theta_{u}$ and $\theta_{v}$ patch together to give a meromorphic connection on $\mathbb{C P}^{1}$ : on the intersection of the two charts we have $d v^{-1}=$ $u^{2} d u^{-1}$ and

$$
\begin{aligned}
\nabla d v^{-1} & =2 u d u \otimes d u^{-1}+u^{2} \nabla d u^{-1}=\frac{2 \rho_{u} \frac{d u}{u}-d \rho_{u}}{\rho_{u}} \otimes\left[u^{2} d u^{-1}\right] \\
& =-\frac{d \rho_{v}}{\rho_{v}} \otimes d v^{-1} .
\end{aligned}
$$

It follows that $\left.d \circ i\right|_{\mathrm{UP}} ^{\circ}=\left.(i \otimes 1) \circ \nabla\right|_{\mathrm{UP}}$. The curvature vanishes on all open sets. The curvature of the line bundle extended across the singular points is given by

$$
\Omega=\bar{\partial} \partial \log \left\|\sigma^{*}\right\|_{Q^{*}}^{2}=\bar{\partial} \partial \log \left|\Delta^{-\frac{1}{6}}\right|^{2}
$$

We obtain

$$
\frac{\Omega}{2 \pi i}=-\sum_{n=1}^{K} \frac{k_{n}}{6} \delta\left(u-u_{n}^{*}\right) d u \wedge d \bar{u}-\frac{10-N_{f}}{6} \delta(v) d v \wedge d \bar{v} .
$$


The equality $\int_{\mathbb{C P}^{1}} i \Omega /(2 \pi)=2$ then follows from $\sum_{n} k_{n}=N_{f}+2$.

REMARK. Under the functor of Fact 7.5 the isomorphism class of $\left(\omega_{\mathbb{C P} 1}^{-1}, \nabla\right)$ corresponds to the monodromy representation of $\pi_{1}(\stackrel{\circ}{\mathrm{UP}})$ on the flat line bundle DET D.

\section{The signature of $\mathrm{Z}$}

In the case that the elliptic fibration $\mathrm{Z} \rightarrow \mathrm{UP}$ has no surface singularities and the singular fibers $E_{u}=\pi^{-1}(u)$ at $u_{1}^{*}, \ldots, u_{N_{f}+2}^{*}$ are nodal curves, the manifold $\mathrm{Z}$ is a smooth four-dimensional manifold with boundary $\partial \mathrm{Z}$. The generalization of Hirzebruch's signature theorem for manifolds with boundary by Atiyah, Patodi, Singer [3] shows that the elliptic signature operator $\mathrm{A}$ on $\mathrm{Z}$ has an analytic index if one imposes APS boundary conditions on $\partial \mathrm{Z}$. The operator $\mathrm{A}$ on $\mathrm{Z}$ is of the form

$$
\mathrm{A}=\sigma\left(\frac{\partial}{\partial|v|}+\mathfrak{D}\right)
$$

near the boundary, where $|v|$ is the inward normal coordinate, $\sigma$ a certain bundle isomorphism, and $\mathfrak{D}$ is the selfadjoint signature operator on the boundary $\partial \mathrm{Z}[\mathbf{2}]$. For the boundary circle $\gamma_{\infty}$ around $u=\infty$, one obtains a three-dimensional manifold $W_{\infty}=\partial \mathrm{Z}$ fibered over a circle. On the boundary component $W_{\infty}$ the selfadjoint signature operator $\mathfrak{D}$ on the differential forms of even degree is

$$
\mathfrak{D}=* d-d *: \quad C^{\infty}\left(W_{\infty}\right) \oplus \Omega^{2}\left(W_{\infty}\right) \rightarrow C^{\infty}\left(W_{\infty}\right) \oplus \Omega^{2}\left(W_{\infty}\right) .
$$

The eigenvalues of the operator $\mathfrak{D}$ can be positive $\lambda_{j}$ or negative $-\mu_{j}$. If we set

$$
\begin{aligned}
\zeta_{|\mathfrak{D}|}(s) & =\sum_{j} \lambda_{j}^{-s}+\sum_{j} \mu_{j}^{-s}, \quad \eta_{\mathfrak{D}}(s)=\sum_{j} \lambda_{j}^{-s}-\sum_{j} \mu_{j}^{-s}, \\
\zeta_{\mathfrak{D}^{2}}(s) & =\sum_{j} \lambda_{j}^{-2 s}+\sum_{j} \mu_{j}^{-2 s}
\end{aligned}
$$

we obtain $\zeta_{\mathfrak{D}^{2}}(s)=\zeta_{|\mathfrak{D}|}(2 s)$. The logarithm of the regularized determinant $\ln \operatorname{det} \mathfrak{D}$ should equal $[\mathbf{2 0}]$

$$
-\left.\frac{d}{d s}\right|_{s=0}\left(\sum_{j} \lambda_{j}^{-s}+(-1)^{s} \sum_{j} \mu_{j}^{-s}\right) .
$$

Making the choice $(-1)^{s}=e^{i \pi s}$ we obtain

$$
\begin{aligned}
\ln \operatorname{det} \mathfrak{D} & =-\left.\frac{d}{d s}\right|_{s=0}\left(\frac{\zeta_{|\mathfrak{D}|}+\eta_{\mathfrak{D}}}{2}+e^{i \pi s}\left(\zeta_{|\mathfrak{D}|}-\eta_{\mathfrak{D}}\right)\right) \\
& =-\zeta_{|\mathfrak{D}|}^{\prime}(0)-\frac{i \pi}{2}\left(\zeta_{|\mathfrak{D}|}(0)-\eta_{\mathfrak{D}}(0)\right) .
\end{aligned}
$$


It follows that $\ln \operatorname{det}|\mathfrak{D}|=-\zeta_{|\mathfrak{D}|}^{\prime}(0)=-\zeta_{\mathfrak{D}^{2}}^{\prime}(2 s) / 2$. Since $\mathfrak{D}$ is a self adjoint operator on the odd-dimensional manifold $W_{\infty}$ it follows that $\zeta_{|\mathfrak{D}|}(0)=0$. We obtain

$$
\frac{\operatorname{det} \mathfrak{D}}{\operatorname{det}|\mathfrak{D}|}=\exp \left(-\frac{i \pi}{2} \eta_{\mathfrak{D}}(0)\right)
$$

It follows:

COROLlary 8.1. The elliptic surface $\mathrm{Z} \rightarrow \mathrm{UP}$ satisfies

$$
\operatorname{sign}(\mathrm{Z})=-\eta_{\mathfrak{D}}(0)=-N_{f}
$$

Proof. For the elliptic surface $\mathrm{Z}$ the canonical class is minus the fiber class. It follows that $c_{1}(Z)^{2}=0$. The main theorem of $[\mathbf{3}]$ when applied to the elliptic surface $\mathrm{Z} \rightarrow \mathrm{UP}$ with $c_{1}^{2}(\mathrm{Z})=0$ yields

$$
\operatorname{sign}(\mathrm{Z})=\int_{\mathrm{Z}} \frac{c_{1}^{2}(\mathrm{Z})}{3}-\eta_{\mathfrak{D}}(0)=-\eta_{\mathfrak{D}}(0) .
$$

On the other hand, the application of Lemma 7.4 yields $\operatorname{sign}(\mathrm{Z})=-N_{f}$.

\section{Conclusion and outlook}

We have shown that the Seiberg-Witten family of elliptic curves defines a four-dimensional, Jacobian elliptic surface $\mathrm{Z} \rightarrow \mathrm{UP}$ with boundary. The signature of $\mathrm{Z}$ is the analytic index of the signature operator on $\mathrm{Z}$ if we impose APS boundary conditions on $\partial Z$. On the other hand, we can compute the index from the logarithmic monodromy of the canonical section of the flat determinant line bundle DET D $\rightarrow$ UP of the signature operator along the fiber of $\mathrm{Z} \rightarrow \mathrm{UP}$. The signature of $\mathrm{Z}$ coincides with the number $N_{f}$ of hypermultiplets in gauge theory.

The identification of the hypermultiplets in the $N=2$ supersymmetric low energy $S U(2)$-Yang-Mills theory with the zero modes of the signature operator on the Jacobian elliptic surface defined by the Seiberg-Witten curve is interesting in the context of string theory. String theory predicts that $N=2$ supersymmetric $S U(2)$-gauge theory in four dimensions emerges from the compactification the type IIB string on a certain $K 3$-fibration $\widetilde{X}_{3} \rightarrow$ $\mathbb{C P}^{1}$. The Calabi-Yau three-fold $\widetilde{X}_{3}$ is determined by the gauge bundle in the heterotic string theory, and in the large base limit becomes $\mathbb{C} \times$ Z . Thus, we conclude that after the compactification the hypermultiplets must arise from the string fields on the internal manifold which are the zero modes of the signature operator.

\section{Acknowledgments}

I would like to thank Isadore Singer and David Morrison for many helpful discussions and a lot of encouragement. 


\section{References}

[1] L. Alvarez-Gaumé, G. Moore, C. Vafa, "Theta functions, modular invariance, and strings." Comm. Math. Phys. 106 (1986), no. 1, 1-40.

[2] M. F. Atiyah, "The Logarithm of the Dedekind $\eta$-Function." Math. Ann. 278 (1987), 335-380.

[3] M. F. Atiyah, V. K. Patodi, I. M. Singer, "Spectral asymmetry and Riemannian geometry. I." Math. Proc. Cambridge Philos. Soc. 77 (1975), 43-69.

[4] M. Bershadsky, S. Cecotti, H. Ooguri, C. Vafa, "Kodaira-Spencer theory of gravity and exact results for quantum string amplitudes" Comm. Math. Phys. 165 (1994), no. $2,311-427$.

[5] J.-M. Bismut, J.-B. Bost, "Fibrés déterminants, métriques de Quillen et dégénérescence des courbes." C. R. Acad. Sci. Paris. Sér. I Math. 307 (1988), no. 7, $317-320$.

[6] J.-M. Bismut, J.-B. Bost, "Fibrés déterminants, métriques de Quillen et dégénérescence des courbes." Acta Math. 165 (1990), no. 1-2, 1-103.

[7] J. M. Bismut, D. S. Freed, "The analysis of elliptic families, I, Metrics and connections on determinant bundles." Comm. Math. Phys. 107 (1986), 103-163.

[8] D. Freed, "Special Kähler manifolds." Comm. Math. Phys. 203 (1999), no. 1, $31-52$.

[9] K. Kodaira, "On compact complex analyric surfaces. I, II, and III." Ann. Math. 71(1960), 111-152, 77(1963), 563-626, 78 (1963), 1-40.

[10] H. Majima, "Asymptotic analysis for integrable connections with irregular singular points." Lecture Notes in Mathematics, 1075. Springer-Verlag, Berlin, 1984.

[11] R. Miranda, "Persson's list of singular fibers for a rational elliptic surface." Math. Z. 205 (1990), 191-211.

[12] R. Miranda, "An Overview of Algebraic surfaces." Algebraic geometry (Ankara, 1995), 157-217, Lecture Notes in Pure and Appl. Math., 193, Dekker, New York, 1997.

[13] R. Miranda, U. Persson, "On extremal rational elliptic surfaces." Math. Z. 193 (1986), 537-558.

[14] M. Nori, "On certain elliptic surfaces with maximal Picard number." Topology 24 (1985), 175-186.

[15] H. K. Nickerson, "On the complex form of the Poincaré lemma." Proc. Amer. Math. Soc. 9 (1958), 183-188.

[16] D. Quillen, "Determinants of Cauchy-Riemann operators on Riemann surfaces." Funktsional. Anal. i Prilozhen. 19 (1985), no. 1, 37-41, 96.

[17] D. B. Ray, I. M. Singer, "Analytic torsion for complex manifolds." Ann. of Math. (2) 98 (1973), 154-177.

[18] R. Seeley, I. M. Singer, "Extending $\bar{\partial}$ to singular Riemann surfaces." J. Geom. Phys. 5 (1988), no. 1, 121-136.

[19] N. Seiberg, E. Witten, "Monopoles, duality and chiral symmetry breaking in $N=2$ supersymmetric QCD." Nucl. Phys. B 431 (1994), 484-550.

[20] I. M. Singer, "Families of Dirac operators with applications to physics. The mathematical heritage of Élie Cartan." Astérisque 1985, Numero Hors Serie, 323-340.

[21] W. I. Weisberger, "Conformal Invariants for Determinants of Laplacians on Riemann Surfaces." Comm. Math. Phys. 112 (1987), no. 4, 633-638.

Department of Mathematics, Colby College, Waterville, Me 04901

E-mail address: andreas.malmendier@colby.edu 
